\title{
A Facile Fabrication of Silver-Coated Copper Nanowires by Galvanic Replacement
}

\author{
Xin He, ${ }^{1}$ Ruihui He, ${ }^{1}$ Qiuming Lan, ${ }^{1}$ Feng Duan, ${ }^{1}$ Jundong Xiao, ${ }^{1}$ Mingxia Song, \\ Mei Zhang, ${ }^{1}$ Yeqing Chen, ${ }^{1}$ and Yang $\mathbf{L i}^{1}$ \\ ${ }^{1}$ School of Applied Physics and Materials, Wuyi University, Jiangmen, Guangdong 529020, China \\ ${ }^{2}$ Collaborative Innovation Center of Atmospheric Environment and Equipment Technology (CICAEET), School of \\ Environmental Science and Engineering, Nanjing University of Information Science and Technology, Nanjing, Jiangsu 201144, China
}

Correspondence should be addressed to Xin He; hexinwyu@126.com

Received 15 March 2016; Revised 20 June 2016; Accepted 27 June 2016

Academic Editor: Vincenzo Baglio

Copyright (C) 2016 Xin He et al. This is an open access article distributed under the Creative Commons Attribution License, which permits unrestricted use, distribution, and reproduction in any medium, provided the original work is properly cited.

\begin{abstract}
We demonstrated a general strategy to fabricate silver-coated copper nanowires by a galvanic replacement, which is guided by the chemical principle that metal ions (silver ions) with a relatively high reduction potential can galvanically etch nanostructure made from a less metal (copper). Well-dispersed and high-yielded copper nanowires were initially synthesized and then introduced into silver-ammonia solution for the growth of silver nanocrystals on the nanowire surfaces under vigorous oscillation. The results of X-ray diffraction, scanning electron microscope, and transmission electron microscope revealed that the silver nanocrystals were uniformly distributed on the copper nanowire surfaces to form $\mathrm{Cu}$-Ag heterostructures. The concentration of silver-ammonia solution and the time of replacement reaction determine the size and density of the silver nanocrystals. Our investigation might pave the way to the synthesis of other bimetallic nanostructures via a facile, fast, and economical route.
\end{abstract}

\section{Introduction}

Bimetallic nanostructures have received great attention due to the compound performance, enhanced activity, and physicochemical stability compared to the individual components. Particularly, the new properties can be obtained owing to the electronic coupling between two different metals, providing more promising applications for the bimetallic nanostructures in catalysis, plasmonics, electronics, and sensing [1-21]. For instance, Stewart et al. fabricated $\mathrm{Cu}-\mathrm{Ag}, \mathrm{Cu}-$ $\mathrm{Au}$, and $\mathrm{Cu}-\mathrm{Pt}$ core-shell nanowires. Compared with pure copper nanowires ( $\mathrm{Cu} \mathrm{NWs}$ ), the resistance to oxidation of the transparent electrode based on these bimetallic nanowires can be effectively improved [7]. Liu et al. have synthesized $\mathrm{Cu}-\mathrm{Pt}$ nanotubes by a galvanic replacement. The nanotubes exhibit superior electrocatalytic activity and stability during oxygen reduction reaction compared with a commercial $\mathrm{Pt} / \mathrm{C}$ catalyst [11]. Jiang et al. reported the synthesis of $\mathrm{Cu}$ Ag hybrid nanowires with strong surface-enhanced Raman scattering sensitivity [20].
As well known, one-dimensional metal nanostructures have been attracting intense attention due to their excellent photoelectric properties [22-25]. Therein, Cu NWs have been widely investigated in the fields of catalysis and photoelectric devices ascribed to the excellent electronic transport properties and low cost in materials [16, 17, 26-31]. However, poor oxidation stability of the $\mathrm{Cu}$ NWs always hinders further practical application. To improve the stability of the $\mathrm{Cu}$ NWs, additional coatings are usually deposited on the NW surface, for example, Au, Ag, Pt, or Pd metallic coatings [16, 17, 3032]. The outer metallic coatings with higher stability not only provide effective passivation against oxidation of the $\mathrm{Cu}$ NWs, but also maintain good conductivity. Among these coating materials, silver is one of competitive candidates since it has better electrical conductivity and stability than copper, and it has lower cost than other noble metals. According to the different combinations of two metals, bimetallic nanostructures can be classified into core-shell structure and heterostructure $[1,2,5,6,8]$. In order to obtain large surface area of the metallic nanowires to further improve the catalysis 


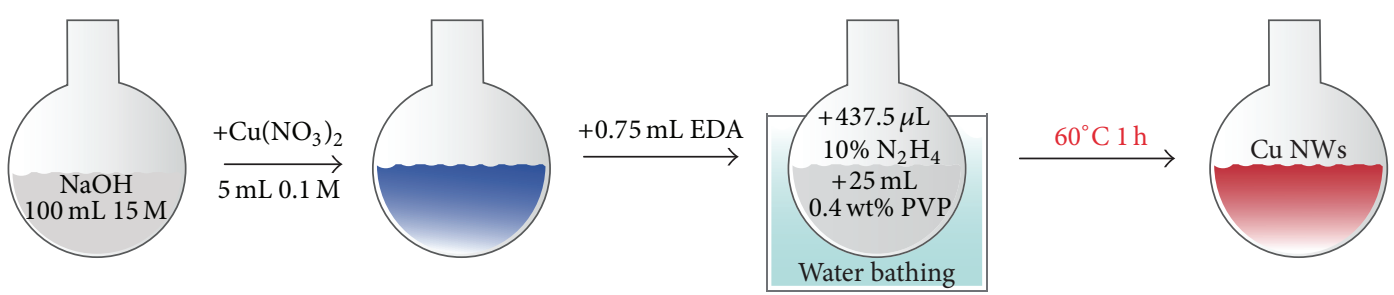

(a)
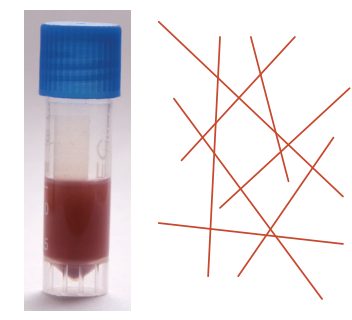

\section{$\underset{+0.025 \mathrm{M} \text { silver-ammonia solution }+}{\stackrel{\text { Vibrating in test tube }}{\longrightarrow}}$ $5 \mathrm{wt} \%$ PVP}

(b)

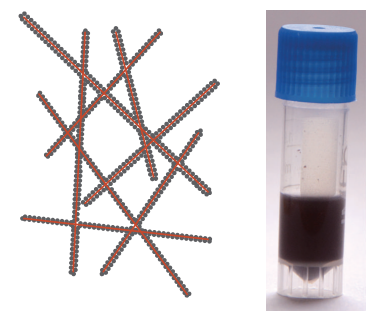

FIGURE 1: Schematic illustration of the fabrication procedure of pure Cu NWs (a) and silver-coated Cu NWs (b), respectively.

activity, silver nanocrystals are usually supposed to be coated on the surface of the $\mathrm{Cu}$ NWs to fabricate silver-copper heterostructure rather than core-shell nanostructure.

Based on this purpose, we developed a facile method to synthesize silver nanocrystals on the surface of highyielded and well-dispersed Cu NWs by galvanic replacement. Although the coating method is usual in the fabrication of bimetals, the experimental parameters need to be accurately controlled in order to synthesize uniform coating. In our work, the replacement reaction took place under vigorous oscillation without any other additives. Silver nanocrystals uniformly grow on the $\mathrm{Cu} \mathrm{NW}$ surface and formed $\mathrm{Cu}$ $\mathrm{Ag}$ heterostructure with $\mathrm{Cu}$ NWs. The surface areas of the bimetallic nanowires are supposed to greatly increase; meanwhile the oxidation of the $\mathrm{Cu}$ NWs is hindered by the coating of the silver nanocrystals. Therefore, this work reveals that more metals with different reduction potentials could facilely synthesize the bimetallic heterostructures by galvanic replacement.

\section{Experimental}

2.1. Synthesis of $\mathrm{Cu} N W s$. Cu NWs were synthesized using a modified chemical reduction [33]. The fabrication procedure is shown in Figure 1(a). Typically, $1.5 \mathrm{~mol}$ sodium hydroxide $(\mathrm{NaOH})$ was dissolved using deionized water of $100 \mathrm{~mL}$ in a flask to obtain $1.5 \mathrm{M} \mathrm{NaOH}$ solution. Then, $5 \mathrm{~mL}$ aqueous solution of copper nitrate $\left(\mathrm{Cu}\left(\mathrm{NO}_{3}\right)_{2} \cdot 3 \mathrm{H}_{2} \mathrm{O}, 0.242 \mathrm{~g}\right.$ in $10 \mathrm{~mL}$ of deionized water) was slowly added to $\mathrm{NaOH}$, yielding a blue transparent solution. The high concentration of $\mathrm{NaOH}$ was required to prevent copper ions from forming copper hydroxide precipitates. $0.75 \mathrm{~mL}$ (99 wt\%) solution of ethylene diamine (EDA) was injected into the blue solution. Following this, $437.5 \mu \mathrm{L}(10 \mathrm{wt} \%)$ hydrazine $\left(\mathrm{N}_{2} \mathrm{H}_{4}\right)$ was dropwise added to the flask to reduce copper ions, and $25 \mathrm{~mL}(0.4 \mathrm{wt} \%)$ solution of poly(vinyl pyrrolidone) (PVP, K30) was added to improve the dispersibility of product. The white precipitates appeared and then the mixture became transparent again as the added amount of $\mathrm{N}_{2} \mathrm{H}_{4}$ increased. The flask was capped and exposed to $60^{\circ} \mathrm{C}$ water bathing for $1 \mathrm{~h}$. Finally, the red product containing $\mathrm{Cu}$ NWs was obtained. $\mathrm{Cu}$ NWs were washed with deionized water containing $1 \mathrm{wt} \%$ PVP and $3 \mathrm{wt} \% \mathrm{~N}_{2} \mathrm{H}_{4}$ several times.

2.2. Synthesis of Silver-Coated $\mathrm{Cu}$ NWs. Silver-coated $\mathrm{Cu}$ NWs were synthesized by a galvanic replacement, and the fabrication procedure is displayed in Figure 1(b). Before replacement, $\mathrm{Cu}$ NWs were washed utilizing ethanol and glacial acid acetic to remove the surfactant and oxide. Silver source derived from silver-ammonia solution, which was prepared according to the following steps. $0.170 \mathrm{~g}$ silver nitrate $\left(\mathrm{AgNO}_{3}\right)$ was dissolved in $25 \mathrm{~mL} \mathrm{H}_{2} \mathrm{O}$, and then ammonia $\left(\mathrm{NH}_{3} \cdot \mathrm{H}_{2} \mathrm{O}\right)$ was dropwise added to $\mathrm{AgNO}_{3}$ solution until white precipitate disappeared, generating silver-ammonia solution. $\mathrm{Cu}$ NWs with a certain quality were introduced into silver-ammonia solution in a test tube along with fierce vibrating. After tens of minutes, the red $\mathrm{Cu}$ NWs turned crimson, demonstrating that the silver-coated $\mathrm{Cu}$ NWs were synthesized. The final products were repeatedly washed by ethanol to remove the redundant $\mathrm{Ag}\left(\mathrm{NH}_{3}\right)_{2}{ }^{+}$ions.

2.3. Characterization. The structures and compositions of the products were characterized using an X-ray diffractometer (XRD) (X'pert Pro MFD) with a $\mathrm{Cu} \mathrm{K \alpha}$ radiation $(\lambda=1.54178 \AA \AA$ ). The morphologies of the nanowires were observed using a field emission scanning electron microscope (FESEM) (Nova ${ }^{\mathrm{TM}}$ NanoSEM 430, FEI), and energy dispersive spectra (EDS) were also conducted on the SEM 430. A transmission electron microscope (TEM) measurement and the element mapping characterization of the nanowires were carried out with JEOL-2100F electron microscope.

\section{Results and Discussion}

Figures 1(a) and 1(b) show a schematic illustration of the fabrication procedure of pure and silver-coated $\mathrm{Cu}$ NWs, 
respectively. During the synthesis process of pure Cu NWs, the aggregation of $\mathrm{Cu}$ NWs can be inhibited by the addition of surfactant PVP. Good dispersibility of the Cu NWs is essential to obtain uniform distribution of silver nanocrystals on the $\mathrm{Cu}$ NW surface.

Figures 2(a) and 2(b) display typical SEM images of the product before replacement reaction, indicating that high-yielded nanowires in a large scale were synthesized. The straight nanowires possess smooth surfaces and uniform diameters, and the length of the nanowires is up to dozens of microns. Figure 2(c) shows diameter distribution histogram of the obtained nanowires, revealing that the average diameter of the nanowires is approximately $107 \mathrm{~nm}$. The corresponding EDS pattern of the nanowires is shown in Figure 2(d), which confirms that main element of the nanowires is $\mathrm{Cu}$, whereas element $\mathrm{Si}$ peak originates from the substrate, and element $\mathrm{C}$ and $\mathrm{O}$ weak peaks derive from the capped surfactant or adsorbed air. Furthermore, XRD measurement was utilized to further explore whether copper had been oxidized in the nanowires. Figure 2(e) displays XRD pattern of the high-yielded nanowires and the standard card of cubic copper (JCPDF number 96-710-1265), respectively. Three obvious and sharp diffraction peaks are detected at $43.2,50.3$, and $74.0^{\circ}$ for the nanowires, which can be assigned to (111), (002), and (022) reflections of face centered cubic structure of metallic copper, respectively. No obvious peaks from copper oxide can be observed, indicating high purity of the $\mathrm{Cu}$ NWs.

Before replacement, the Cu NWs were repeatedly washed by ethanol and glacial acid acetic and then dried in vacuum oven at $60^{\circ} \mathrm{C}$. The dried $\mathrm{Cu}$ NWs with a certain quality were redispersed in ethanol and glacial acid acetic and then introduced into various concentrated silver-ammonia solution to keep molar ratio of $\mathrm{Cu}$ and $\mathrm{Ag} 2: 1$ and 1:2. The mixed dispersions were persistently vibrated in a test tube to synthesize the silver-coated $\mathrm{Cu}$ NWs. Two products were named as samples A and B, respectively. The silver ions possess a relatively high reduction potential compared to copper ions, which can lead to a galvanic replacement between silver ions and copper at ambient condition. Therefore, in our work, it is believed that metallic $\mathrm{Cu}$ NWs can reduce the $\mathrm{Ag}\left(\mathrm{NH}_{3}\right)_{2}{ }^{+}$ ions adsorbed on their surfaces, leading to the formation of metallic silver.

Figures 3(a) and 3(b) present representative SEM images of samples A and B, respectively. The inset of Figure 3(b) is a magnified image. Several nanocrystals are observed on the smooth surface of $\mathrm{Cu}$ NWs after galvanic replacement, which can be ascribed to silver nanocrystals. Once $\mathrm{Ag}\left(\mathrm{NH}_{3}\right)_{2}{ }^{+}$ions were reduced to metallic silver on $\mathrm{Cu}$ NW surface, a large strain can be generated due to the lattice mismatch between silver and copper at the interface, providing driving force to form silver nanocrystals [5]. As the concentration of silverammonia solution increased, the surface roughness of the $\mathrm{Cu}$ NWs is greatly improved, and the numbers and diameters of the nanocrystals are increased. Compared to the pure $\mathrm{Cu}$ NWs, the surface areas of the bimetallic nanowires thus are effectively enlarged, potentially promoting the catalytic activities. Furthermore, the nanocrystals are uniformly distributed on the surface of the $\mathrm{Cu}$ NWs via galvanic replacement under vigorous oscillation, and no individual nanocrystals can be observed in solution. It indicates that the replacement of $\mathrm{Ag}\left(\mathrm{NH}_{3}\right)_{2}{ }^{+}$by metallic $\mathrm{Cu}$ just takes place on the surface of the $\mathrm{Cu}$ NWs. The oscillation offers lots of opportunities for the collisions between $\mathrm{Ag}\left(\mathrm{NH}_{3}\right)_{2}{ }^{+}$ions and $\mathrm{Cu} \mathrm{NW}$ surface, resulting in uniform distribution of silver nanocrystals. The individual nanocrystals cannot be synthesized in solution due to the absence of any other reducing agent. Moreover, if silver nitrate substitutes for silver-ammonia solution in this work, the replacement reaction would take place very quickly. The mixed solution turned black in several seconds, and uneven coatings were observed. It is believed that the $\mathrm{Ag}\left(\mathrm{NH}_{3}\right)_{2}{ }^{+}$ chelate ions are beneficial to a mild replacement reaction, generating uniform coatings of the nanocrystals on the $\mathrm{Cu}$ NW surfaces.

Figures 3(c) and 3(d) display EDS patterns of the nanocrystals-coated $\mathrm{Cu}$ NWs in samples $\mathrm{A}$ and $\mathrm{B}$, suggesting that the main elements of the nanowires are $\mathrm{Cu}$ and $\mathrm{Ag}$, and the content of element $\mathrm{Ag}$ in sample B is higher than that in sample A. However, the contents of element $\mathrm{O}$ in two samples are not markedly increased compared to the pure $\mathrm{Cu}$ NWs, demonstrating that the nanocrystals on the nanowire surfaces can effectively hinder the oxidation of the $\mathrm{Cu} \mathrm{NWs}$. Moreover, the atomic ratios of element $\mathrm{Cu}$ to $\mathrm{Ag}$ in two samples are larger than the predefined ratios of 2 and 0.5. The surface of $\mathrm{Cu}$ NWs provides active sites for the replacement reaction. Once the silver nanocrystals occupy all active sites on the surface, the further galvanic replacement almost stops. The redundant $\mathrm{Ag}\left(\mathrm{NH}_{3}\right)_{2}^{+}$ions in the solution are hard to be further reduced to metallic silver due to the lack of any reducing agent in the solution, leading to a high atomic ratio of element $\mathrm{Cu}$ to $\mathrm{Ag}$ in two samples. If we extended the reaction time for sample $\mathrm{A}$, the sizes and numbers of the silver nanocrystals could further increase.

Figure 3(e) shows XRD patterns of samples A and B, and the standard cards of cubic copper and silver are provided for reference. In two patterns of samples A and B, the diffraction peaks located at 43.2 and $50.4^{\circ}$ can be assigned to (111) and (002) reflections of face centered cubic structure of metallic copper (JCPDF number 96-710-1265). The diffraction peak originated from silver cannot be observed in the pattern of sample A due to low loading of silver nanocrystals on the $\mathrm{Cu} \mathrm{NW}$ surface. However, as the content of silver increased, the peak at $38.0^{\circ}$ appeared, which matches well with the (111) reflection of face centered cubic structure of metallic silver (JCPDF number 01-087-0597). It is worthy to note that the peak positions of metallic copper in sample B do not shift as silver nanocrystals formed, revealing that the nanostructure of the $\mathrm{Cu}$ NWs cannot be changed by silver nanocrystals. The obtained product is heterostructure of $\mathrm{Cu}$ NWs and silver nanocrystals, rather than bimetallic alloy.

Figure 4(a) presents a typical TEM image of several silver-coated $\mathrm{Cu}$ NWs. It can be clearly observed that many small black dots are uniformly attached on the surface of every single nanowire. Particularly, more dots are located at the junctions of the straight nanowires. Combined with the above discussion, the attached dots are silver nanocrystals. Figure 4(b) shows TEM image of single silver-coated Cu NW, indicating a uniform distribution of the silver nanocrystals 


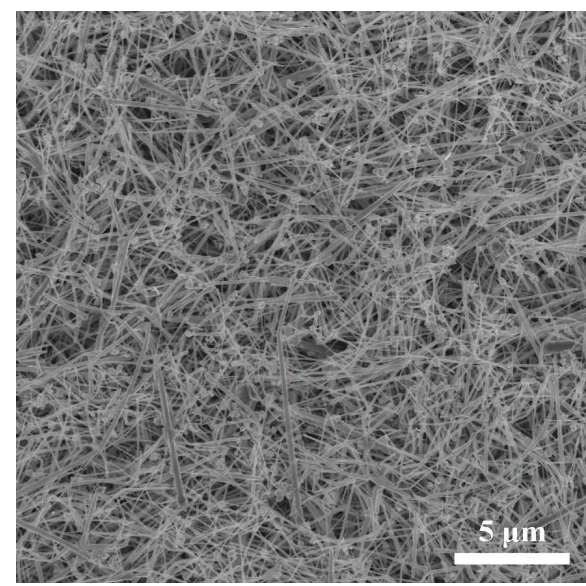

(a)

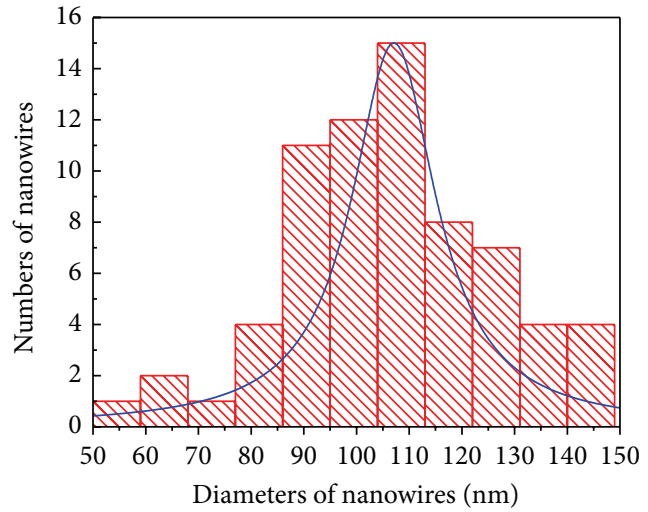

(c)

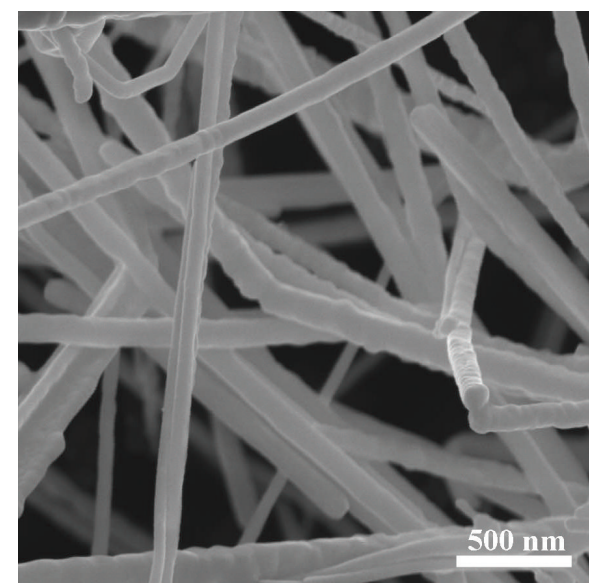

(b)

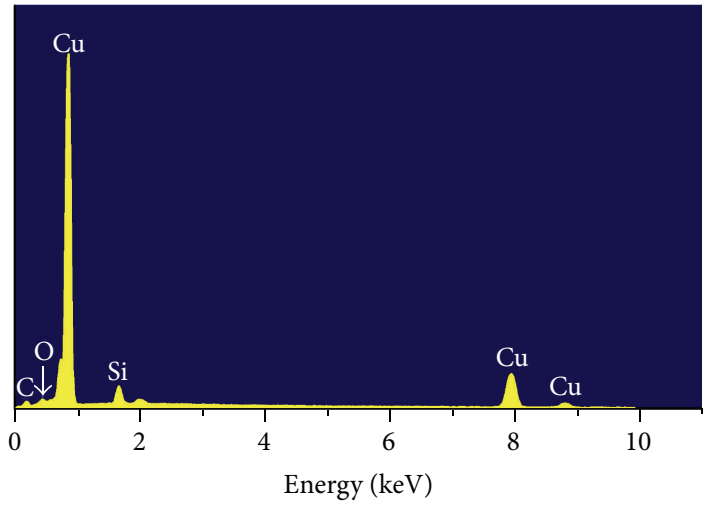

(d)

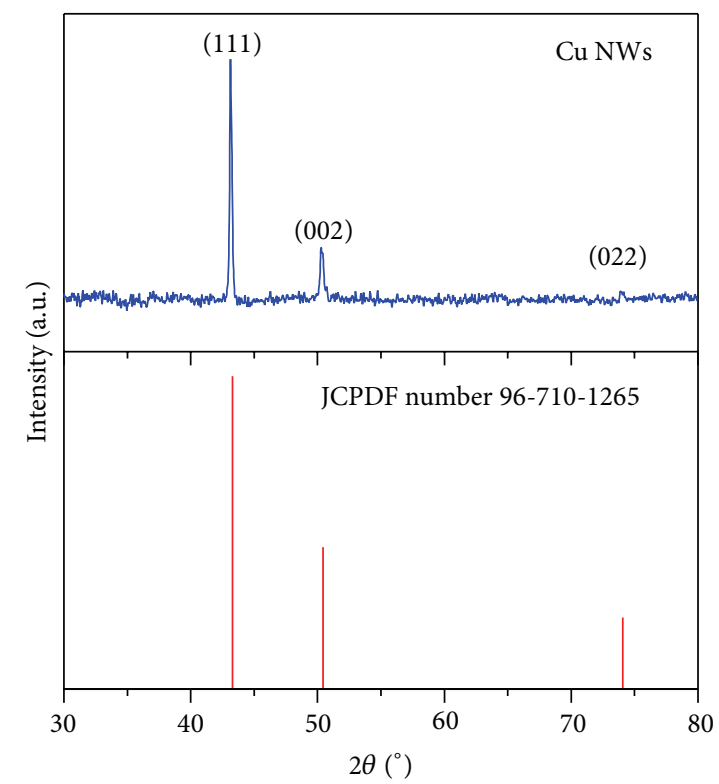

(e)

FIGURE 2: SEM images of high-yielded Cu NWs with low (a) and high (b) magnification; (c) diameter distribution histogram of the nanowires; (d) corresponding EDS pattern of the nanowires; (e) XRD pattern of the Cu NWs, and the PDF card of cubic copper (JCPDF number 96710-1265) is provided for reference. 


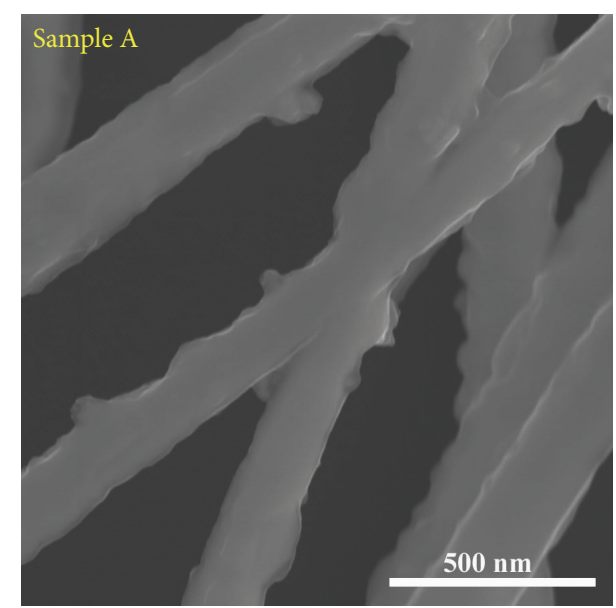

(a)

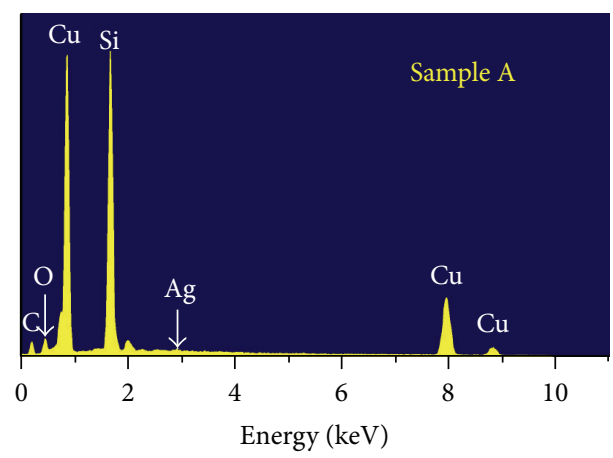

(c)

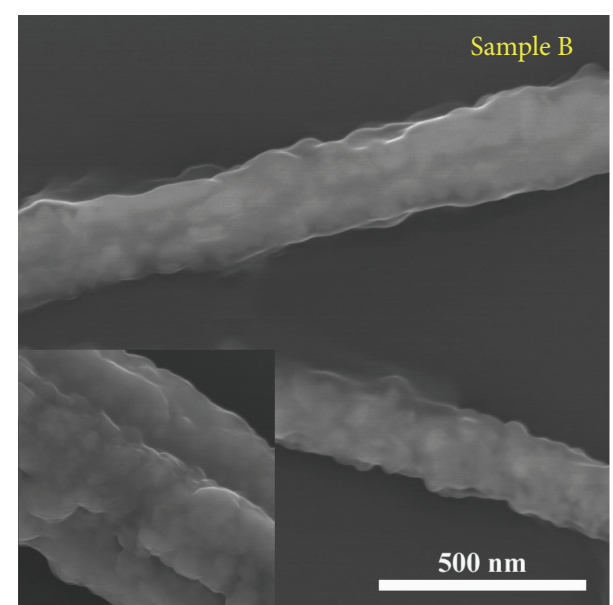

(b)

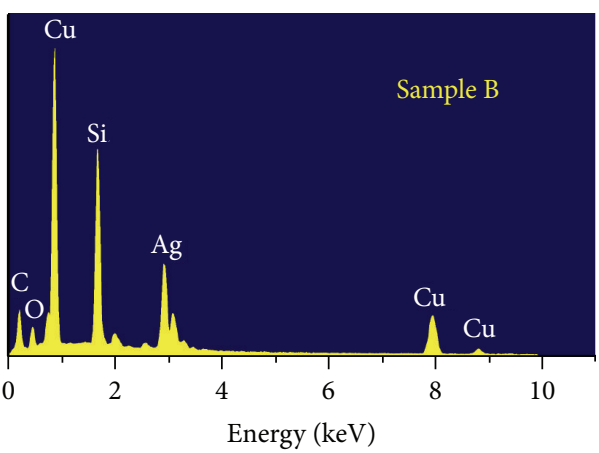

(d)

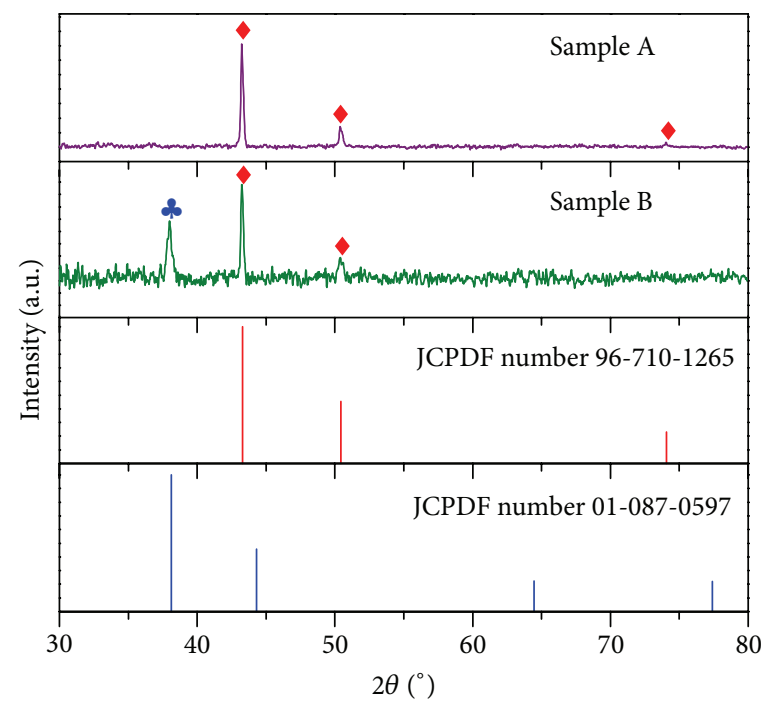

(e)

Figure 3: SEM images of the silver-coated Cu NWs for samples A (a) and B (b); the inset of (b) is a magnified image; EDS patterns of the silver-coated Cu nanowires for samples A (c) and B (d), respectively; (e) XRD patterns of the silver-coated Cu nanowires for samples A and $\mathrm{B}$, and the PDF cards of cubic copper (red line, JCPDF number 96-710-1265) and silver (blue line, JCPDF number 01-087-0597) are provided for reference. The red rhombus represents the reflection of face centered cubic structure of metallic copper (JCPDF number 96-710-1265), while the blue star represents reflection of face centered cubic structure of metallic silver (JCPDF number 01-087-0597). 


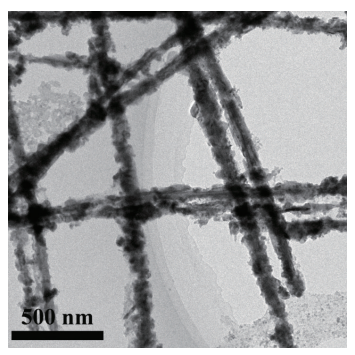

(a)

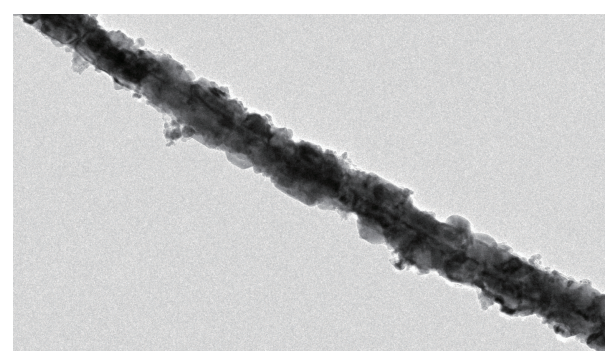

(b)

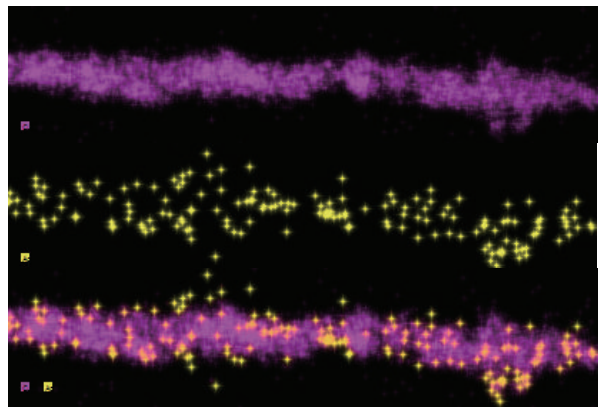

(c)

FIGURE 4: (a) TEM image of the silver-coated Cu NWs; (b) TEM image of single silver-coated Cu NW and its element mapping images (c); the purple and yellow dots represent elements copper and silver, respectively.

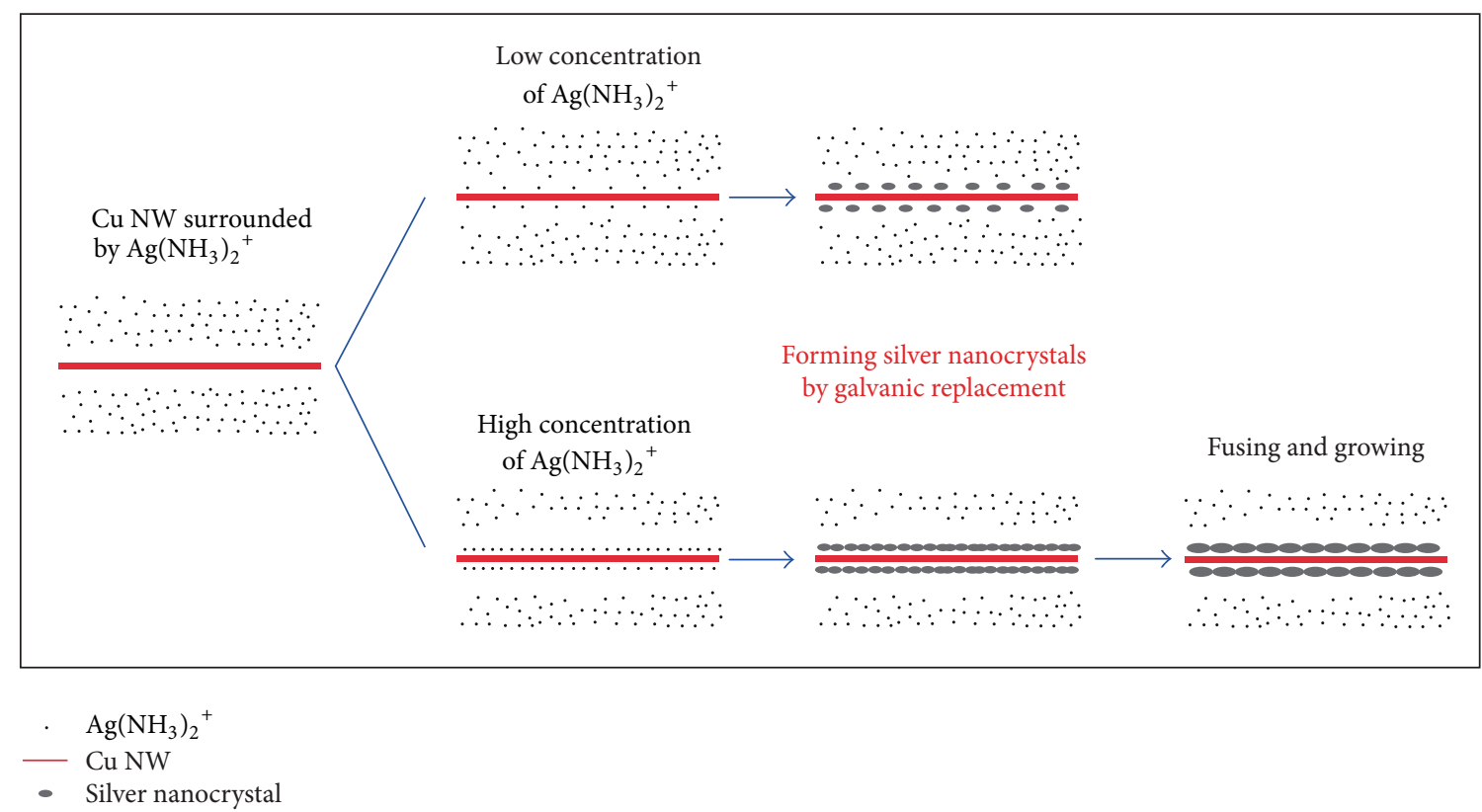

FIGURE 5: Schematic diagram of a possible growth process of silver-coated Cu NWs.

on the $\mathrm{Cu} \mathrm{NW}$ surface, and initial smooth surface of $\mathrm{Cu}$ NW appears to be relatively rough. The element mapping images of one selected silver-coated $\mathrm{Cu} \mathrm{NW}$ are displayed in Figure 4(c), in which the purple and yellow dots represent elements $\mathrm{Cu}$ and $\mathrm{Ag}$, respectively. The images reveal that the straight nanowire consists of principally copper, and silver nanocrystals are decorated on the surface of the nanowire. The TEM results are consistent with the above SEM analysis.

Figure 5 illustrates a schematic diagram of the synthesis of silver-coated $\mathrm{Cu} \mathrm{NWs}$ with low and high $\mathrm{Ag}\left(\mathrm{NH}_{3}\right)_{2}{ }^{+}$ concentration, respectively. The $\mathrm{Cu}$ NWs were initially introduced into silver-ammonia solution, thus surrounded by many $\mathrm{Ag}\left(\mathrm{NH}_{3}\right)_{2}{ }^{+}$ions. The adsorbed $\mathrm{Ag}\left(\mathrm{NH}_{3}\right)_{2}{ }^{+}$ions on the surface of $\mathrm{Cu}$ NWs could be reduced to metallic silver via galvanic replacement. The large strain generated at the $\mathrm{Cu}$-Ag interface can provide driving force to form silver nanocrystals. The numbers and sizes of the nanocrystals synthesized with a high concentration of $\mathrm{Ag}\left(\mathrm{NH}_{3}\right)_{2}{ }^{+}$ions are larger than those that are synthesized with a low concentration of $\mathrm{Ag}\left(\mathrm{NH}_{3}\right)_{2}{ }^{+}$ions. It can be considered that high concentration of silver source provides more possibility for adsorption or collision between the $\mathrm{Ag}\left(\mathrm{NH}_{3}\right)_{2}{ }^{+}$ions and $\mathrm{Cu} \mathrm{NW}$ surface during the same reaction time, resulting in more silver nanocrystals formed. In this case, a lot of crystal boundaries are generated among the nanocrystals. The small nanocrystals can fuse and further grow to form one with a larger size. Certainly, if the reaction time is further extended, the numbers and sizes of the nanocrystals with a low concentration of $\mathrm{Ag}\left(\mathrm{NH}_{3}\right)_{2}{ }^{+}$ions can also be considerably increased.

\section{Conclusion}

In summary, uniform coating of the silver nanocrystals on the $\mathrm{Cu}$ NW surface could be synthesized by facile galvanic replacement under vigorous oscillation. Good dispersibility of the pure $\mathrm{Cu}$ NWs is important to improve the distribution of the silver nanocrystals. The silver ions are reduced to 
metallic silver on the surface of the Cu NWs to generate large strain at the $\mathrm{Cu}$-Ag interface. The strain can provide driving force to form silver nanocrystals. The resistance of oxidation to the $\mathrm{Cu}$ NWs can be effectively improved by depositing silver nanocrystals. Our work suggests a facile, fast, and lowcost way to synthesize desired heterostructures consisting of different metals.

\section{Competing Interests}

There are no competing interests related to this paper.

\section{Acknowledgments}

This work was supported by the National Natural Science Foundation of China (Grants nos. 61307026 and 11305091); the Natural Science Foundation of Guangdong Province (Grants nos. 2014A030313621 and 2015A030313645); the Frontier and Key Technological Innovation Special Foundation of Guangdong Province (Grant no. 2014B090915001).

\section{References}

[1] S. Chen, S. V. Jenkins, J. Tao, Y. Zhu, and J. Chen, "Anisotropic seeded growth of $\mathrm{Cu}-\mathrm{M}(\mathrm{M}=\mathrm{Au}, \mathrm{Pt}$, or $\mathrm{Pd})$ bimetallic nanorods with tunable optical and catalytic properties," The Journal of Physical Chemistry C, vol. 117, no. 17, pp. 8924-8932, 2013.

[2] A. Muzikansky, P. Nanikashvili, J. Grinblat, and D. Zitoun, "Ag dewetting in $\mathrm{Cu@Ag} \mathrm{monodisperse} \mathrm{core-shell} \mathrm{nanoparticles,"}$ The Journal of Physical Chemistry C, vol. 117, no. 6, pp. 30933100, 2013.

[3] J. Zhang, X. Xu, C. Yang, F. Yang, and X. Yang, "Colorimetric iodide recognition and sensing by citrate-stabilized core/shell Cu@Au nanoparticles," Analytical Chemistry, vol. 83, no. 10, pp. 3911-3917, 2011.

[4] L. Jiang, W. Zhu, C. Wang et al., "Preparation of hollow $\mathrm{Ag} / \mathrm{Pt}$ heterostructures on $\mathrm{TiO}_{2}$ nanowires and their catalytic properties," Applied Catalysis B: Environmental, vol. 180, pp. 344-350, 2016.

[5] M. Han, S. Liu, L. Zhang et al., "Synthesis of octopus-tentaclelike $\mathrm{Cu}$ nanowire-Ag nanocrystals heterostructures and their enhanced electrocatalytic performance for oxygen reduction reaction," ACS Applied Materials and Interfaces, vol. 4, no. 12, pp. 6654-6660, 2012.

[6] S. Khanal, A. Spitale, N. Bhattarai et al., "Synthesis, characterization, and growth simulations of Cu-Pt bimetallic nanoclusters," Beilstein Journal of Nanotechnology, vol. 5, pp. 1371-1379, 2014.

[7] I. E. Stewart, S. Ye, Z. Chen, P. F. Flowers, and B. J. Wiley, "Synthesis of $\mathrm{Cu}-\mathrm{Ag}, \mathrm{Cu}-\mathrm{Au}$, and $\mathrm{Cu}-\mathrm{Pt}$ core-shell nanowires and their use in transparent conducting films," Chemistry of Materials, vol. 27, no. 22, pp. 7788-7794, 2015.

[8] A. Sarkar and A. Manthiram, "Synthesis of Pt@Cu core-shell nanoparticles by galvanic displacement of $\mathrm{Cu}$ by $\mathrm{Pt}^{4+}$ ions and their application as electrocatalysts for oxygen reduction reaction in fuel cells," The Journal of Physical Chemistry $C$, vol. 114, no. 10, pp. 4725-4732, 2010.

[9] A. Bansal, J. S. Sekhon, and S. S. Verma, "Scattering efficiency and LSPR tunability of bimetallic $\mathrm{Ag}$, $\mathrm{Au}$, and $\mathrm{Cu}$ nanoparticles," Plasmonics, vol. 9, no. 1, pp. 143-150, 2014.
[10] H. Zhang, M. Haba, M. Okumura, T. Akita, S. Hashimoto, and $\mathrm{N}$. Toshima, "Novel formation of $\mathrm{Ag} / \mathrm{Au}$ bimetallic nanoparticles by physical mixture of monometallic nanoparticles in dispersions and their application to catalysts for aerobic glucose oxidation," Langmuir, vol. 29, no. 33, pp. 10330-10339, 2013.

[11] J. Liu, C. $\mathrm{Xu}, \mathrm{C}$. Liu et al., "Impact of $\mathrm{Cu}-\mathrm{Pt}$ nanotubes with a high degree of alloying on electro-catalytic activity toward oxygen reduction reaction," Electrochimica Acta, vol. 152, pp. 425-432, 2015.

[12] S. Zeng, L. Nguyen, F. Cheng, L. Liu, Y. Yu, and F. Tao, "Surface structure and chemistry of $\mathrm{Pt} / \mathrm{Cu} / \mathrm{Pt}\left(\begin{array}{lll}1 & 1 & 1\end{array}\right)$ near surface alloy model catalyst in CO," Applied Surface Science, vol. 320, pp. 225230, 2014.

[13] J. Tymoczko, F. Calle-Vallejo, V. Čolić, W. Schuhmann, and A. S. Bandarenka, "Evaluation of the electrochemical stability of model Cu-Pt(111) near-surface alloy catalysts," Electrochimica Acta, vol. 179, pp. 469-474, 2015.

[14] J. Duan, Q. Tang, H. Zhang, Y. Meng, L. Yu, and P. Yang, "Counter electrode electrocatalysts from one-dimensional coaxial alloy nanowires for efficient dye-sensitized solar cells," Journal of Power Sources, vol. 302, pp. 361-368, 2016.

[15] S. Guo, X. Zhang, W. Zhu et al., "Nanocatalyst superior to Pt for oxygen reduction reactions: the case of core/shell $\mathrm{Ag}(\mathrm{Au}) / \mathrm{CuPd}$ nanoparticles," Journal of the American Chemical Society, vol. 136, no. 42, pp. 15026-15033, 2014.

[16] Z. Chen, S. Ye, A. R. Wilson, Y.-C. Ha, and B. J. Wiley, "Optically transparent hydrogen evolution catalysts made from networks of copper-platinum core-shell nanowires," Energy and Environmental Science, vol. 7, no. 4, pp. 1461-1467, 2014.

[17] S. M. Alia, B. S. Pivovar, and Y. Yan, "Platinum-coated copper nanowires with high activity for hydrogen oxidation reaction in base," Journal of the American Chemical Society, vol. 135, no. 36, pp. 13473-13478, 2013.

[18] S. W. T. Price, J. D. Speed, P. Kannan, and A. E. Russell, "Exploring the first steps in core-shell electrocatalyst preparation: in situ characterization of the underpotential deposition of $\mathrm{Cu}$ on supported Au nanoparticles," Journal of the American Chemical Society, vol. 133, no. 48, pp. 19448-19458, 2011.

[19] Y. Sun, F. Zhang, L. Xu, Z. Yin, and X. Song, "Roughnesscontrolled copper nanowires and $\mathrm{Cu}$ nanowires-Ag heterostructures: synthesis and their enhanced catalysis," Journal of Materials Chemistry A, vol. 2, no. 43, pp. 18583-18592, 2014.

[20] Z. Jiang, Y. Tian, S. Ding, J. Wen, and C. Wang, "Facile synthesis of $\mathrm{Cu}-\mathrm{Ag}$ hybrid nanowires with strong surface-enhanced Raman scattering sensitivity," CrystEngComm, vol. 18, no. 7, pp. 1200-1206, 2016.

[21] Y. Wei, S. Chen, Y. Lin, Z. Yang, and L. Liu, "Cu-Ag core-shell nanowires for electronic skin with a petal molded microstructure," Journal of Materials Chemistry C, vol. 3, no. 37, pp. 95949602, 2015.

[22] Y. Xia, K. Sun, and J. Ouyang, "Solution-processed metallic conducting polymer films as transparent electrode of optoelectronic devices," Advanced Materials, vol. 24, no. 18, pp. 24362440, 2012.

[23] D. S. Hecht, L. Hu, and G. Irvin, "Emerging transparent electrodes based on thin films of carbon nanotubes, graphene, and metallic nanostructures," Advanced Materials, vol. 23, no. 13, pp. 1482-1513, 2011.

[24] J. Li, J. Liang, X. Jian, W. Hu, J. Li, and Q. Pei, "A flexible and transparent thin film heater based on a silver nanowire/heatresistant polymer composite," Macromolecular Materials and Engineering, vol. 299, no. 11, pp. 1403-1409, 2014. 
[25] M.-G. Kang, T. Xu, H. J. Park, X. Luo, and L. J. Guo, "Efficiency enhancement of organic solar cells using transparent plasmonic Ag nanowire electrodes," Advanced Materials, vol. 22, no. 39, pp. 4378-4383, 2010.

[26] R. Wang and H. Ruan, "Synthesis of copper nanowires and its application to flexible transparent electrode," Journal of Alloys and Compounds, vol. 656, pp. 936-943, 2016.

[27] H. Guo, N. Lin, Y. Chen et al., "Copper nanowires as fully transparent conductive electrodes," Scientific Reports, vol. 3, article 2323, 2013.

[28] S. Li, Y. Chen, L. Huang, and D. Pan, "Large-scale synthesis of well-dispersed copper nanowires in an electric pressure cooker and their application in transparent and conductive networks," Inorganic Chemistry, vol. 53, no. 9, pp. 4440-4444, 2014.

[29] S. Ye, A. R. Rathmell, I. E. Stewart et al., "A rapid synthesis of high aspect ratio copper nanowires for high-performance transparent conducting films," Chemical Communications, vol. 50, no. 20, pp. 2562-2564, 2014.

[30] D. Yang, J. Carpena-Núñez, L. F. Fonseca, A. Biaggi-Labiosa, and G. W. Hunter, "Shape-controlled synthesis of palladium and copper superlattice nanowires for high-stability hydrogen sensors," Scientific Reports, vol. 4, article 3773, 2014.

[31] R. P. Chauhan and P. Rana, "Nickel ion induced modification in the electrical conductivity of $\mathrm{Cu}$ nanowires," Radiation Measurements, vol. 83, pp. 43-46, 2015.

[32] B. W. An, E.-J. Gwak, K. Kim et al., "Stretchable, transparent electrodes as wearable heaters using nanotrough networks of metallic glasses with superior mechanical properties and thermal stability," Nano Letters, vol. 16, no. 1, pp. 471-478, 2016.

[33] Y. Chang, M. L. Lye, and H. C. Zeng, "Large-scale synthesis of high-quality ultralong copper nanowires," Langmuir, vol. 21, no. 9, pp. 3746-3748, 2005. 

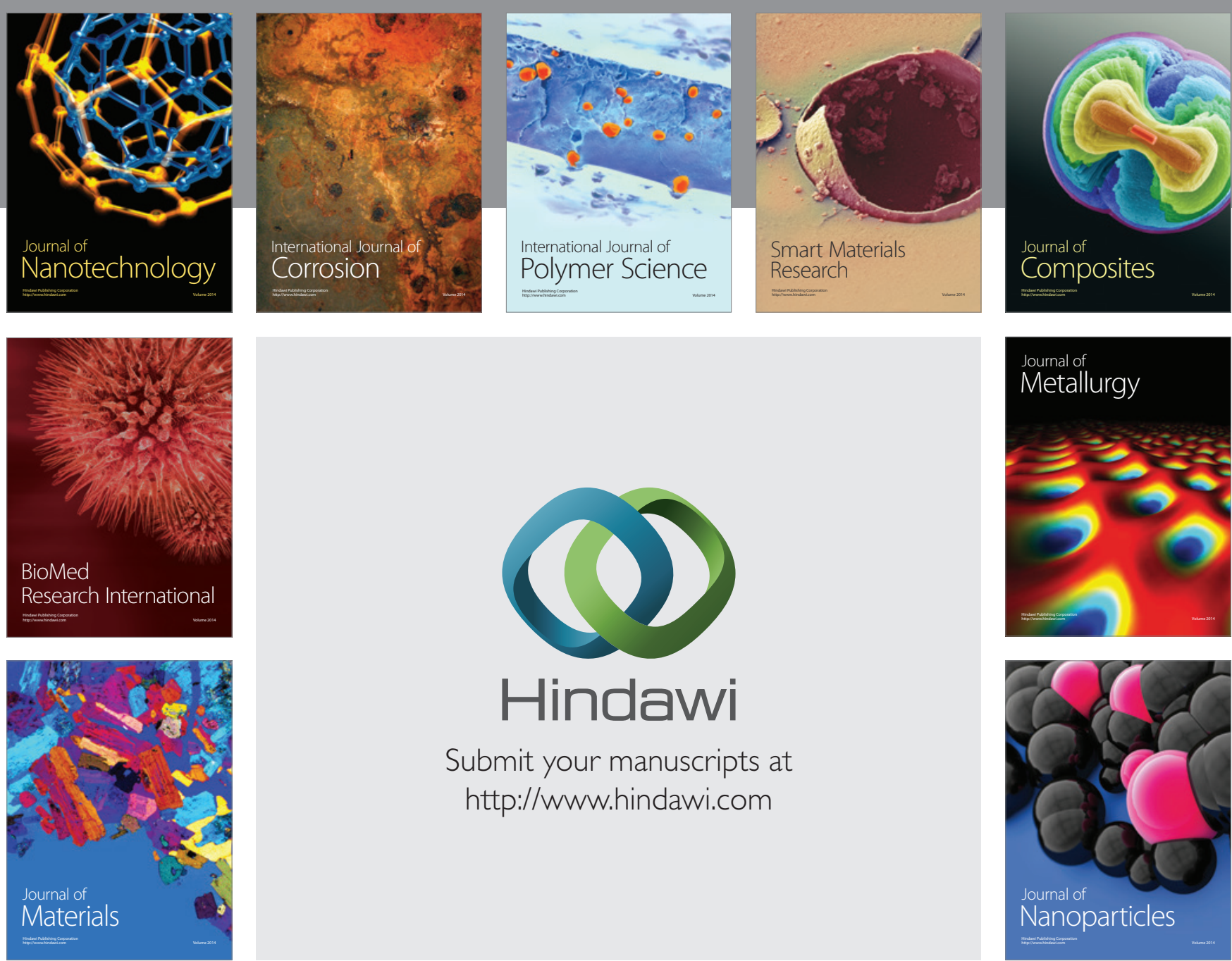

\section{Hindawi}

Submit your manuscripts at

http://www.hindawi.com

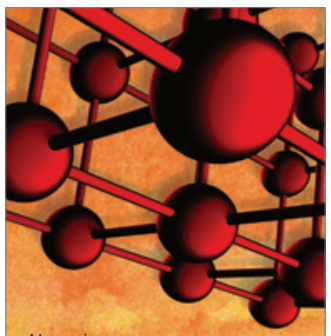

Materials Science and Engineering
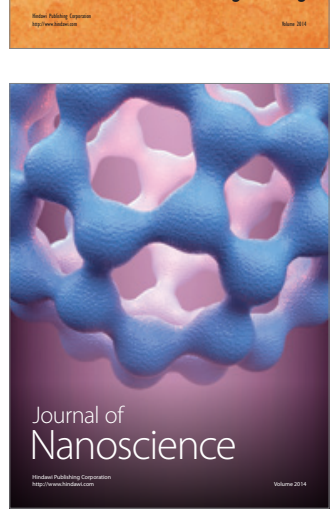
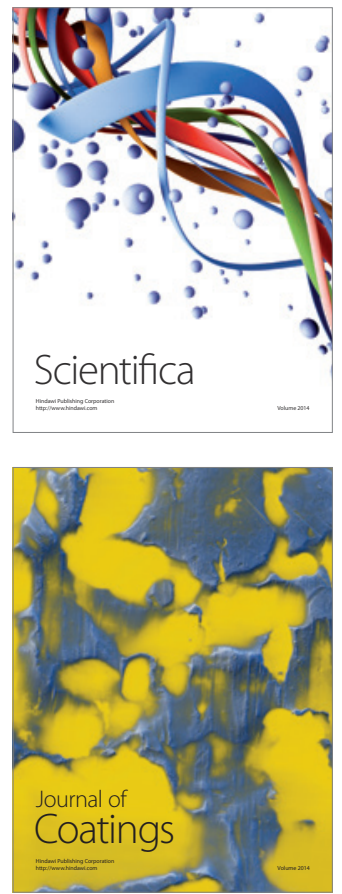
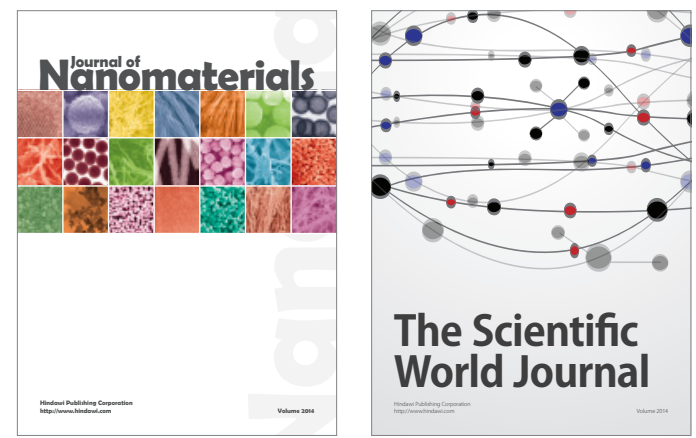

The Scientific World Journal
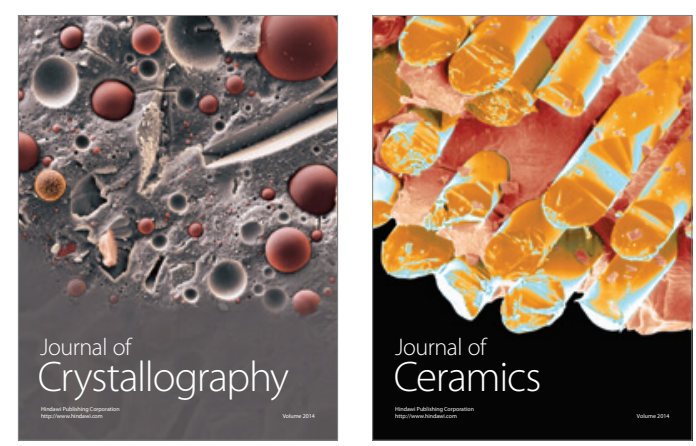
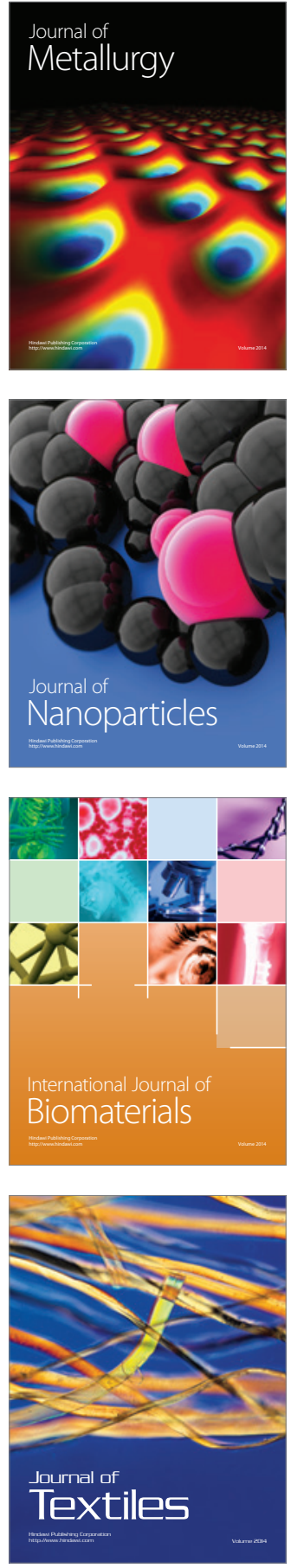